\title{
HOW TO FIND BL LAC OBJECTS IN THE RASS
}

\author{
P. NASS \\ Max-Planck-Institut für Extraterrestrische Physik, Germany
}

\section{Introduction}

The correlation of an X-ray survey like the ROSAT All-Sky Survey (RASS) with optical surveys like the Hamburg Quasar Survey (HQS) can significantly increase the number of known BL Lac objects.
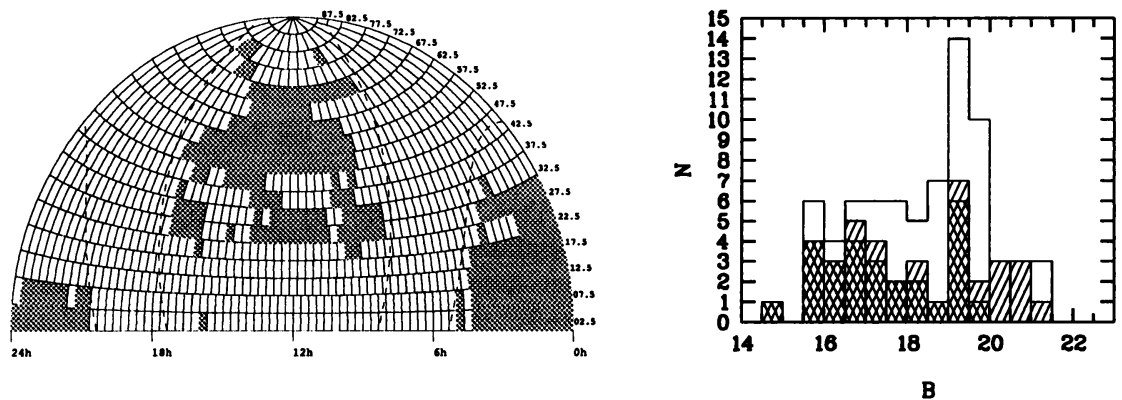

Figure 1. Left: In the studied area (shaded) we identified 35 new BL Lac objects (Nass et al. 1996) ; 43 BL Lac objects were previously known, from which we excluded 4 star-like objects. The ROSAT All-Sky Survey (RASS) was correlated with these 216 fields from the Hamburg Quasar Survey (HQS) during an ongoing AGN identification program. To select BL Lac candidates we used the criterion $\log f_{X} / f_{B}>1.3$. During the follow-up spectroscopy of the first subsample of $\log f_{X} / f_{B}>1.3$ candidates, $40 \%$ of them proved to be BL Lac objects. The efficiency rate will be higher after the complete identification, because the remaining candidate objects have higher $\log f_{X} / f_{B}$ and for a given X-ray flux BL Lac objects tend to be optically fainter than other types of AGN. Right: Among the newly identified BL Lac objects (blank) are more optically faint ones than among the previously known BL Lac objects of which 27 (crossed) were and 12 (striped) were not detected in the RASS. The decrease beyond $\mathrm{B}=19$ is probably caused by the platelimit and the difficulty to detect a featureless spectrum of such a weak object. Follow-up observations of BL Lac candidates with 'empty' RASS error circles on HQS plates could enhace the number of BL Lac objects fainter than $B=19.5$. 
$\mathrm{X}$-ray sources with the highest ratios of X-ray to optical flux are primarily BL Lac objects. Therefore, by selecting high $\log \left(f_{x} / f_{B}\right)$ objects, very good BL Lac candidates can be chosen. This is a radio independent search technique which is very important for the weaker BL Lac objects which do not enter common radio catalogues with thresholds of 25 to $40 \mathrm{mJy}$. However, cross-correlation with more sensitive radio catalogs helps to optimize the candidate list and saves optical observing time at large telescopes (very important for faint objects). RBL-like objects cannot be discovered with this technique but with the combined X-ray/radio/optical method which consists of the calculation of the energy indices of $\mathrm{X}$-ray sources with a radio counterpart. By taking spectra of candidates which populate the typical BL Lac area in the $\alpha_{R O}-\alpha_{O X}$ diagram one can simply identify new BL Lac objects.
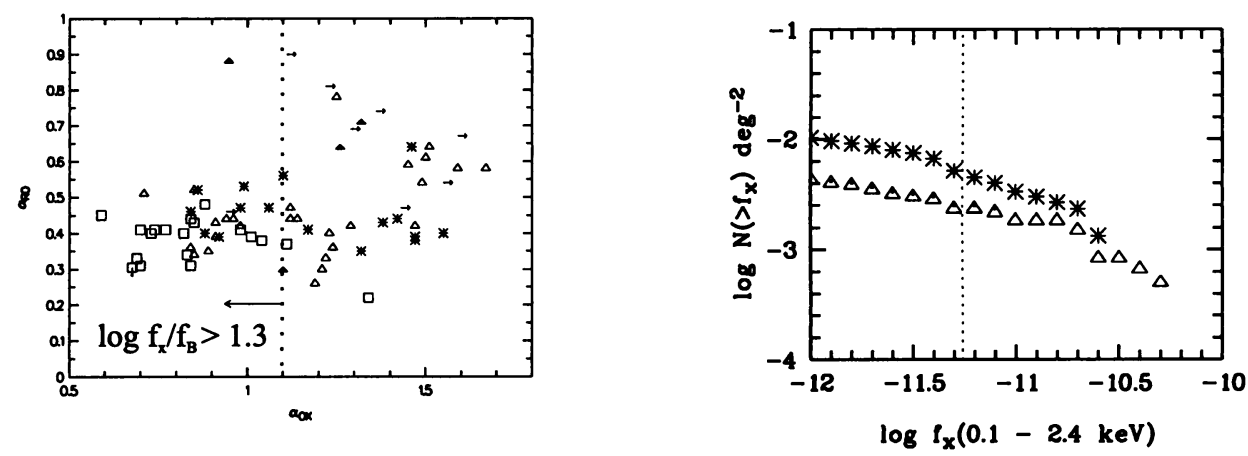

Figure 2. Colour-Colour-Diagram(left): The 39 previously known BL Lac objects can be found in the typical areas: XBLs populate a "box" in the lower left corner and RBLs are aligned along a diagonal at higher $\alpha_{O X}$ and higher $\alpha_{R O} .27$ objects (open triangles) were detected in the RASS, 12 not. 4 (filled triangles) of these 12 objects were detected in pointed PSPC observations. For the remaining 8 objects (arrows), we used the upper limits from the RASS. Most new objects (asterisks) populate the XBL region, those without entry in radio catalogues(empty boxes) at lower $\alpha_{R O} . \log \mathrm{N}-\log$ $\mathrm{S}$ (right): The obtained surface density is in accordance with previous studies. However, we suspect more BL Lac objects at lower X-ray fluxes which remained undiscovered due to previously used search techniques.

\section{Conclusions}

The combination of X-ray, optical, and radio surveys is a very efficient means for the discovery of new BL Lac objects in X-ray surveys like the RASS. Our results indicate that about $200 \mathrm{BL}$ Lac objects can be found among the high $\log f_{x} / f_{B}$ BL Lac candidates in the studied area. A larger sample of BL Lac objects is needed to ensure that selection effects did not influence the existing $\log \mathrm{N}-\log \mathrm{S}$ distributions and BL Lac models. Par- 
ticularly, the reason for the seperation of the whole BL Lac class into XBLs and RBLs is important. Will we still see it in a larger BL Lac sample? Additionally, further studies can be made, e.g., our environmental studies of BL Lac objects with possibly extended X-ray emission.

\section{References}

Nass P., Bade, N. ,Kollgaard, R. I. et al.,1996, Astron.Astrophys.,309,419 